\title{
REGISTRATION OF TRANSCRANIAL MAGNETIC STIMULATION, A VISUALIZATION TOOL FOR BRAIN FUNCTIONS
}

\author{
Quentin Noirhomme ${ }^{1}$, Eduardo Romero ${ }^{1}$, Olivier Cuisenaire ${ }^{2}$, Matthieu Ferrant ${ }^{4}$, \\ Yves Vandermeeren ${ }^{3}$, Etienne Olivier ${ }^{3}$ and Benôt Macq ${ }^{1}$ \\ ${ }^{1}$ Laboratoire de Télécommunications et Télédetection \\ Université catholique de Louvain \\ Batiment Stevin - 2, place du Levant \\ B-1348 Louvain La Neuve, Belgium \\ ${ }^{2}$ Swiss Federal Institute of Technology (EPFL), \\ Signal Processing Laboratory, Lausanne. \\ ${ }^{3}$ Neurophysiology Laboratory, Université catholique de Louvain \\ ${ }^{4}$ General Electric, Paris \\ \{noirhomme, romero, macq\}atele.ucl.ac.be, matthieu.ferrant@med.ge.com, \\ \{yves.vandermeeren, olivier\}@nefy.ucl.ac.be, olivier.cuisenaire@epfl.ch
}

\begin{abstract}
We describe a method for the registration of functional brain datas acquired with transcranial magnetic stimulation (TMS) on MRI brain images. TMS is a non-invasive method largely used in the study of brain functions. For the registration process we acquire 150 points of the patient's scalp with magnetic-field digitizer. Then, we minimize the mean square distance between those points and the segmented scalp surface drawn from MR image. The distance to the scalp surface is computed with the help of a 3D Euclidean distance transformation. For each stimulation, the position of the TMS device is acquired with the digitizer. The registration transformation is applied to the TMS coordinate in order to map TMS data and anatomical information. The results show that the method is precise $(4 \mathrm{~mm})$ and reproducible $(1 \mathrm{~mm})$.
\end{abstract}

\section{INTRODUCTION}

In their daily exploration of brain functions, specialists can use a large set of tools. While Magnetic Resonance Imaging (MRI) is nowadays the most common method used for anatomical studies of central nervous system, a variety of techniques including PET, functional MRI (fMRI), electroencephalogram (EEG), magnetoencephalogram (MEG) or transcranial magnetic stimulation (TMS) provide the complementary functional information. In this paper, we will concentrate on the TMS, a non-invasive method that allows to obtain functional mapping of cortex regions by exciting or inhibiting specific area. A pulsed magnetic field creates enough current flow in the brain to modulate complex and measurable responses. This technique has been widely used in the study of cortical physiology, brain plasticity, brain excitability in neurologic disorders or brain therapy [4].

Modern neurophysiological studies require functional informations to be correlated with anatomical localization. On the one hand, PET and fMRI are imaging techniques and can therefore be registered using classical image to image registration methods. On the other hand, EEG, MEG and TMS are essentially providing information on the surface of the scalp and require a registration between the MRI image and the physical space. PET, fMRI and TMS give different but complementary informations about the structure of the brain [4].
Different methods are proposed to register the TMS data on the anatomy. Bastings [1] uses six fiducial points that are marked with adhesive MRI-visible label. The 3Dcoordinates of these points can be determined with a digitizer. Therefore they are a common reference which can be used to register the TMS data and the MRI with one algorithm of distance minimization, in the least square sense. In Ettinger [3], the TMS device and the position of the head is tracked with the help of three linear cameras which localize flashing IR LEDS and a laser scanner that digitizes the scalp surface. Five flashing IR LEDS are placed on the subject's head and two on the TMS device. The MR image is registered with the laser space and the LEDS give information on the head motion. Potts [7] describes an evolution of the system that does not require the laser. Wang [9] has developped a method to register EEG and MEG. He samples 400 points on the head with a magnetic digitizer in order to digitize head surface. Then the surface is aligned on the MRI using Pelizzari's algorthm [6].

In this paper we present an automated registration method that does not require MR-visible markers, laser or camera. It uses a simple acquisition technique and is easily repeatable. Section 2 describes the method. Section 3 presents our validation tests and the comparison with other methods. 


\section{METHOD}

Our method can be divided in four steps: acquisition of the MR image, segmentation of the image, TMS and registration. The order of steps is not important. We can realize the TMS before acquiring the MR image but, of course, the registration is always at the end of the process.

After acquisition of the image of the patient's head, the brain and cortical surfaces are segmented using a generic semi-automatic watershed segmentation algorithm [8]. This requires the user to place a few markers inside and outside the object of interest, then the procedure extends those markers automatically to the whole 3D image. If needed, the procedure can work iteratively with the user placing additional markers in the most difficult areas. Meshed surfaces are then generated using the marching cubes algorithm [5] applied on the segmented image. The original voxel values are restored on the edges of objects so that partial volumes and local gradients can be taken into account to produce a surface with sub-pixel accuracy. The coordinates of the segmented head and scalp are given by the MRI coordinate system.

During the TMS, we use a figure- 8 shaped coil to stimulate the cortex. A magnetic-field digitizer (Polhemus Isotrak II) with two receivers is used to acquire the TMS coordinates. The first receiver is fixed on the patient's forehead to track the move of the head and the second is a hand held stylus. In this way, the patients'head doesn't need to be fixed, and it is, therefore, more comfortable. Finally a surface electrode is placed on the muscle of interest to record the responses to the stimulations.

Before to start the TMS, we use the digitizer to acquire about 150 points of the scalp to registrate the coordinates of the TMS with the coordinates of the MR images. These points follow the shape of the scalp as illustrated in Figure 1 (left). This first phase is very fast and takes only a couple of minutes.

The position of the TMS coil is tracked with the hand held stylus. For each stimulation we take three mesures: one at the coil center and the other two in the coil plane.

The registration of the TMS data and MR images is ini-
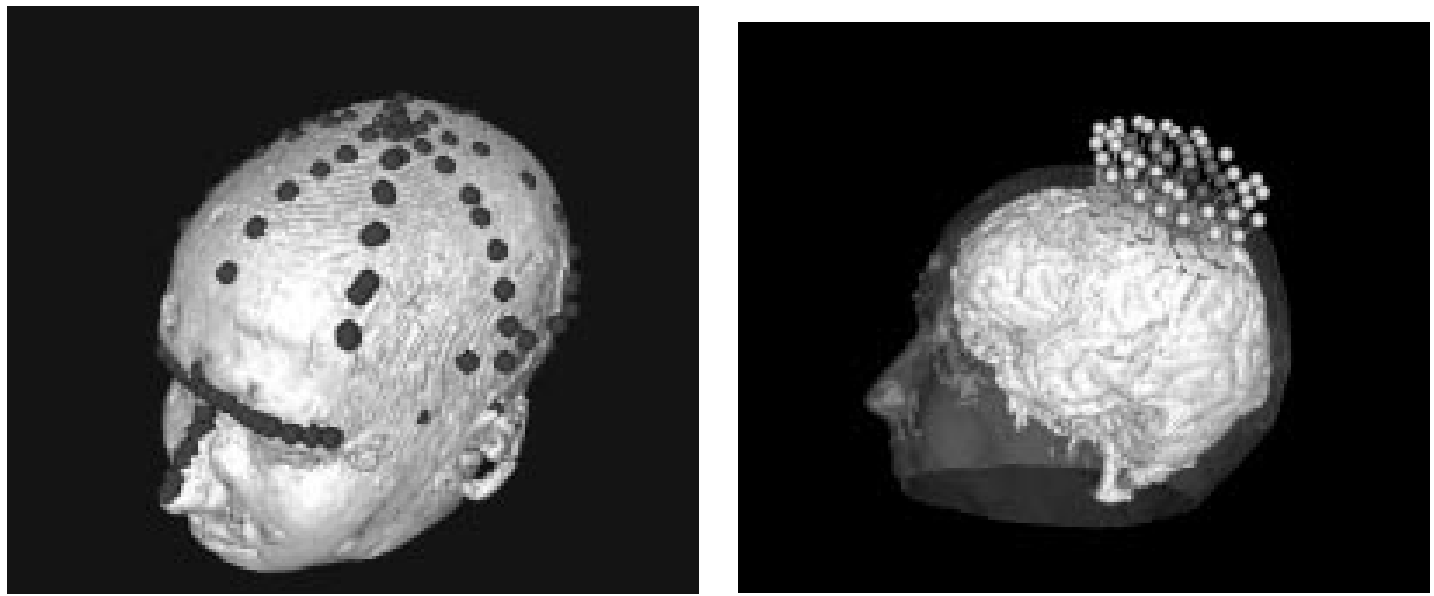

Fig. 1. Left: scalp surface with digitized points for the registration; Right the magnet position and orientation 

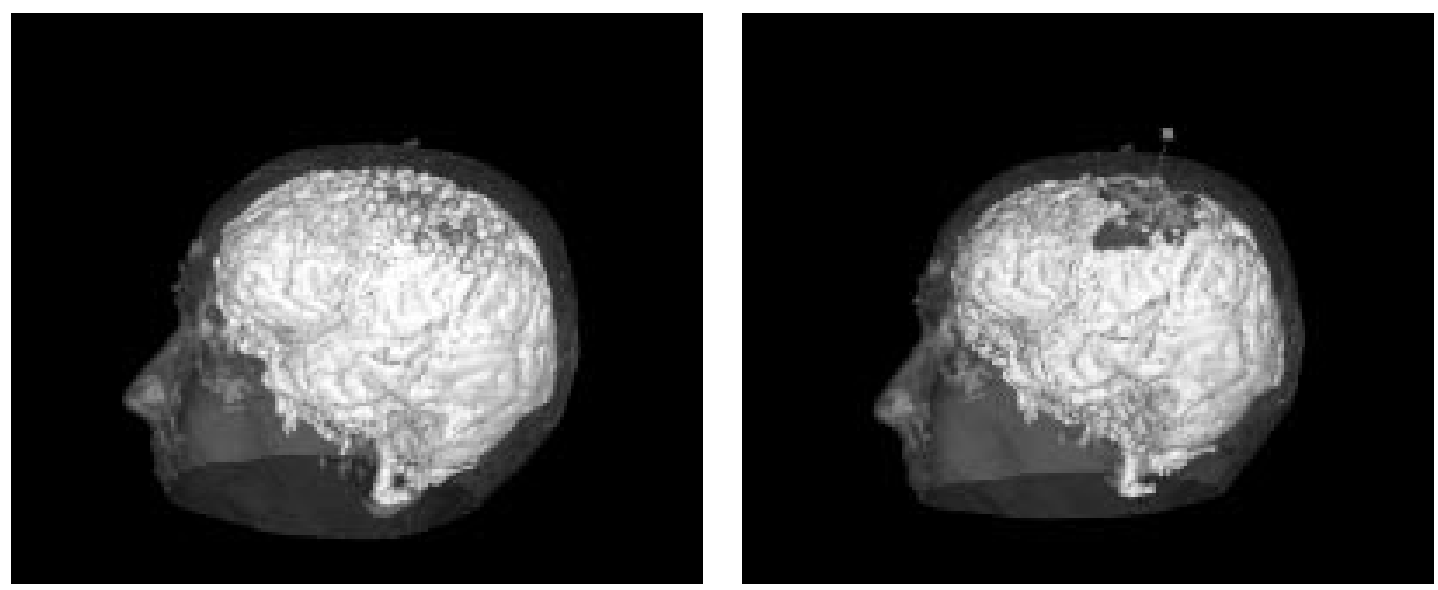

Fig. 2. Left: spheres projection on brain surface; Right interpolated brain map and centers of gravity

\section{RESULTS AND DISCUSSION}

We assess the precision and accuracy of our method on multiple levels, from the registration algorithm on its own to the whole system including the mapping on the scalp and brain surfaces of the activation patterns.

First we consider the registration algorithm on its own. A random sample of 200 points from the scalp surface computed from the MRI was used as a synthetic set of digitized points. Arbitrary rigid transformations were applied on those points and try to recover the correct registration transform. The algorithm converges for any translation and for any rotation of less than $30^{\circ}$. The precision of the registration was evaluated from the distance between the original points and the points to which both arbitrary and registration transforms were applied. This test was performed for 100 different arbitrary transforms from within the convergence domain of the algorithm, obtaining a mean distance of $0.17 \mathrm{~mm} \pm 0.3 \mathrm{~mm}$ and a maximum distance of $0.31 \mathrm{~mm}$.

The second validation uses real digitized points. 5 sets of points were acquired and the registrations were performed. Table 1 shows the resulting mean square distances between the registered points and the scalp surface, i.e. the matching criterion used by the registration algorithm.In the 5 cases, the points to surface distance is about $1 \mathrm{~mm}$.

For routine use, the above measure is the only one available to assess the accuracy of the registration, but it is not a sufficient proof of precision. Indeed, for roughly spherical surfaces, a small surface to surface distance could occur despite of a large rotational error. Therefore, we acquired an image with MRI-visible markers whose location were also digitized during the 5 trials. Table 2 shows the distances between the registered markers and their location picked from the MRI. The average error of is about $3.8 \mathrm{~mm}$.

Finally, the reproducibility of the whole procedure was evaluated. For this purpose, we compute the location of the centers of gravity ( $\mathrm{Cog}$ ) of a stimulation of the motor cortex resulting from 4 different registrations. This assesses both the registration accuracy itself and its influence on the projection of activations onto the scalp and brain surfaces. The accuracy of the method is evaluated from the distance between the $\mathrm{CoG}$ and the mean CoG found by the method. The mean distances were about $1.3 \mathrm{~mm}$ to the scalp and about $1 \mathrm{~mm}$ to the Brain. Also, the location of the CoG is found more accurately on the brain than on the scalp surface.

Precision and accuracy should be compared to the $4.4 \mathrm{~mm}$ precision cited by Bastings [1] and the $5 \mathrm{~mm}$ achieved by Wang [9]. Wang also cites a root-mean-square distance of $2.7 \mathrm{~mm}$ between its two surfaces, we found $0.17 \mathrm{~mm} \pm 0.3 \mathrm{~mm}$. To the best of our knowledge, Ettinger and Potts $[3,7]$ do not provide a quantitative assessment of the precision of their method.

The time required by the procedure can be divided into two parts. The processing required only once per patient and the processing required for every stimulation. First, once for each patient one needs to acquire a MRI and to process these data. The processing mostly requires segmentation of the head and cortical surfaces. This typically requires about 10 minutes of combined user-interaction and CPU time. Secondly, for every stimulation one needs to digitize the scalp surface and perform the registration. While digitizing the scalp has previously been reported to take up to an hour [9], we found out that scalp points can be digitized semi-continuously by scanning the scalp surface along a few lines. In our experience, scalp digitization requires between 1 and 3 minutes. Digitizing the coil locations requires approximately 30 seconds per location. Registration itself requires an initial manual placement, the computation of the distance transformation and the minimization of the mean square distance. The whole process is performed in a couple of minutes. 
Table 1. Assessment of our method: mean square distance $\left(\mathrm{mm}^{2}\right)$ from digitized points to scalp surface

\begin{tabular}{|c|c|c|c|c|c|c|}
\hline & Trial 1 & Trial 2 & Trial 3 & Trial 4 & Trial 5 & Mean \\
\hline Mean sq. dist. & 1.223 & 1.042 & 0.921 & 0.975 & 1.132 & 1.059 \\
\hline
\end{tabular}

Table 2. Assessment of our method: distance to fiducial markers $(\mathrm{mm})$

\begin{tabular}{ccccccc}
\hline & Trial 1 & Trial 2 & Trial 3 & Trial 4 & Trial 5 & Mean \\
\hline Point 1 & 4.709 & 4.399 & 4.907 & 7.419 & 3.425 & $\mathbf{4 . 9 7 2}$ \\
Point 2 & 4.959 & 4.142 & 3.667 & 6.508 & 2.377 & $\mathbf{4 . 3 3 1}$ \\
Point 3 & 3.562 & 2.394 & 1.779 & 1.879 & 2.746 & $\mathbf{2 . 4 7 2}$ \\
Point 4 & 7.662 & 5.327 & 5.021 & 3.359 & 2.990 & $\mathbf{4 . 8 7 2}$ \\
Point 5 & 2.498 & 1.861 & 2.551 & 1.109 & 2.886 & $\mathbf{2 . 1 8 1}$ \\
All Points & 4.678 & 3.625 & 3.585 & 4.055 & 2.885 & $\mathbf{3 . 7 6 5}$ \\
\hline
\end{tabular}

\section{CONCLUSION}

We have described a method to register functional brain data acquired with TMS in the physical space on MR image. This method is easy to employ and does not need of specific tools except a MF digitizer. The registration process is precise and reproducible. It needs only a MR image and digitized points of the scalp surface, and can be used for other physical space to image registration like EEG and MEG applications. The registration algorithm has several advantages when compared to previous methods: it does not require MRI-visible markers and no restraint on the patient head.

Furthermore, the TMS and the acquisition of MR image have to be done nor in a short laps of time neither in a specific order. Finally, its accuracy and precision are comparable to or better than those reported by other authors. Registrations can be reproduced with an error of no more than $1 \mathrm{~mm}$, which is approximately the MRI pixel size. Comparison with fiducial markers indicates a precision of approximately $4 \mathrm{~mm}$.

\section{REFERENCES}

[1] P.E Bastings, H.D. Gage, J.P. Greenberg, G. Hammond, L. Hernandez, P. Santago, C.A. Hamilton, D.M. Moody, K.D. Singh, P.E. Ricci, T.P. Pons and D.C. Good, " Co-registration of cortical magnetic stimulation and functional magnetic resonance imaging," NeuroReport 9, 1998, pp 1941-1946.

[2] O. Cuisenaire, "Distance transformation, fast algorithms and applications to medical image processing," Ph.D. Thesis, October 1999, Université catholique de Louvain, B-1348 Louvain-la-Neuve, Belgium.
[3] G.J. Ettinger, M.E. Leventon, W.E.L. Grimson, R. Kikinis, V. Gugino, W. Cote, L. Sprung, L. Aglio, M. Shenton, G. Potts and E. Alexander, "Experimentation with a Transactionscranial Magnetic Stimulation System for Functional Brain Mapping," Proc. CVRMed/MRCAS'97, Grenoble, France.

[4] M. Hallet, "Transcranial magnetic stimulation and the human brain”, Nature, No 406, July 2000.

[5] Lorensen W.E. and Cline H.E., "Marching cubes, a high resolution $3 \mathrm{~d}$ surface contruction algorithm," Computer Graphics, 1997, pp 163-169.

[6] C.A. Pelizzari, G.T.Y. Chen, D.R. Spelbring, R.R. Weichselbaum and C.T. Chen, "Accurate threedimensional registration of CT, PET and/or MR images of the brain," J. Comput. Assist. Tomogr., No 13 1989, pp 20-26.

[7] G.F. Potts , L.D. Gugino, M.E. Leventon, W.E.L. Grimson, R. Kikinis, W. Cote, E. Alexander, J.E. Anderson, G.J. Ettinger, L. Aglio and M.E. Shenton, "Visual Hemifield Mapping Using Transactionscranial Magnetic Stimulation Coregistered with Cortical Surfaces Derived from Magnetic Resonance Images," J. Clin. Neurophysiology, 15, 1998, pp. 344-350.

[8] J.P. Thiran, V. Warscotte and B. Macq, "A queue based region growing algorithm for accurate segmentation of multi-dimensional images," Signal Processing, 60, 1997, pp. 1-10

[9] B. Wang, C. Toro, T.A. Zeffiro and M. Hallet, "Head surface digitization and registration: a method for mapping positions on the head onto magnetic resonance images" Brain Topography, 6, 1994, pp. 185192. 